\title{
Preparation and Properties of Poly(acrylic acid)-Stabilized Magnetite Nanoparticles
}

\author{
Yi-Jun Lin ${ }^{a}$, Leeyih Wang ${ }^{b^{*}}$, J.G. Lin $^{\mathrm{b}}$, Y.Y. Huang ${ }^{\mathrm{b}}$, and Wen-Yen Chiu ${ }^{\mathrm{a}}$ \\ ${ }^{a}$ Department of Chemical Engineering, National Taiwan University, Taipei, Taiwan \\ ${ }^{\mathrm{b}}$ Center for Condensed Matter Sciences, National Taiwan University, Taipei, Taiwan
}

\begin{abstract}
This work presents an efficient way of preparing stable aqueous magnetite dispersion. TEM, XRD, light scattering, Zeta-potential, and magnetization techniques were adopted to characterize the poly(acrylic acid)-coated $\mathrm{Fe}_{3} \mathrm{O}_{4}$ nanoparticles. Experimental results indicated that the average particle size was around $10 \mathrm{~nm}$, with these nanoparticles exhibiting a superparamagnetic behavior.
\end{abstract}

Keywords: Magnetite; nanoparticle; ferrofluid; poly(acrylic acid)

\section{Introduction}

Ferrofluid characteristically consists of both magnetic and fluid properties and has found a diverse range of applications, such as in audio devices, inertia dampers, stepper motors, sensors, and vacuum seals [1]. However, a limitation of ferrofluid is that the nanoparticles have a large ratio of surface-area to volume, likely causing particle aggregation to reduce their surface energy. Consequently, how to prevent the aggregation between the nanoparticles during the synthesis and coating process has received considerable attention. Stable ferrofluid can be prepared by adsorption of stabilizing agents on the magnetite particle surface after a synthetic process. The feasibility of preparing magnetic fluids using anionic or nonionic surfactants as dispersing agents has been examined [2]. This study presents a novel procedure to synthesize an extremely stable, water-based $\mathrm{Fe}_{3} \mathrm{O}_{4}$ ferrofluid.

\section{Experimental}

Iron( II ) chloride tetrahydrate (99\%), iron(III) chloride hexahydrate $(97 \%)$, ammonium hydroxide $\left(28 \% \mathrm{NH}_{3}\right.$ in water, double distilled), and poly(acrylic acid) (PAA; $\left.M_{\mathrm{w}} \sim 2000\right)$ were purchased from ACROS and used as supply. $\mathrm{Fe}_{3} \mathrm{O}_{4}$ particles were prepared by treating 2.0 equiv. of $\mathrm{FeCl}_{2} \cdot 4 \mathrm{H}_{2} \mathrm{O}$ with 3.0 equiv. of $\mathrm{FeCl}_{3} \cdot 6 \mathrm{H}_{2} \mathrm{O}$ in $50 \mathrm{ml}$ of deionized water at a stirring rate of $300 \mathrm{rpm}$ under room temperature, followed by adding $100 \mathrm{ml}$ of ammonium hydroxide. After a reaction of time of around six minutes, the resulting particles were washed three times with 5\% $\mathrm{NH}_{4} \mathrm{OH}$ solution by the magnetic decantation method. A mixture of $3 \mathrm{~g}$ of PAA solution and the precipitate was then heated to $90^{\circ} \mathrm{C}$ for 4 minutes under continuous stirring. Next, excess polyelectrolyte was removed by three centrifugation $(10000 \times \mathrm{g}) / \mathrm{wash} /$ redispersion cycles. The particle sizes and morphologies of the magnetic nanoparticles were also examined by transmission electron microscopy (Hitachi H-7100). Addtionally, X-ray diffraction measurements with monochromatic $\mathrm{CuK}_{\alpha}$ radiation were taken to investigate the crystal structure of the particle. The colloidal stability and particle size distributions were characterized by Zeta-potential (Zetasizer 3000HS-Advanced) instrument and dynamic light scattering (Malvern 4700), and the magnetization of the samples was measured by PPMS (Quantum Design) at $15 \mathrm{~K}$.

\section{Results and discussion}

The magnetic fluid containing $\mathrm{Fe}_{3} \mathrm{O}_{4}$ nanoparticles was prepared by the chemical coprecipitation of ferric and ferrous salts in alkaline medium, based on Reimers' procedure [3], followed by treatment with an aqueous solution of low-molecular-weight PAA. Importantly, using PAA as a dispersing agent leads to a situation in which the resulting ferrofluid possesses an excellent colloidal stability towards dilution with water and the processes of centrifugation-redispersion and freeze-thaw cycles. Moreover, electrophoretic mobility measurements provided

* Corresponding author. Tel: +886-2-3366-5276; fax: +886-2-2369-6221; E-mail: leewang@ccms.ntu.edu.tw 
qualitative proof of the stability of ferrofluid. A negative sign of the zeta potential was observed, in which the value was $-37.6 \mathrm{mV}$. This observation implied the successful adsorption of PAA on particle surface, which provides both electrostatic and steric repulsion against particles' flocculation.

Fig. 1 displays X-ray diffraction pattern of the PAA-stabilized powders. Three main-peaks appeared at $2 \theta$ angles of 30.1, 35.4, 43.0, indicating that the nanoparticles can be identified as $\mathrm{Fe}_{3} \mathrm{O}_{4}$; no other crystalline phases were observed. Moreover, the size of nanoparticles was determined from the diffraction peak by extending the use of the Scherrer's equation [4]. In doing so, the crystallite size of PAA-coated $\mathrm{Fe}_{3} \mathrm{O}_{4}$ particle was calculated to be approximately $9.9 \mathrm{~nm}$.

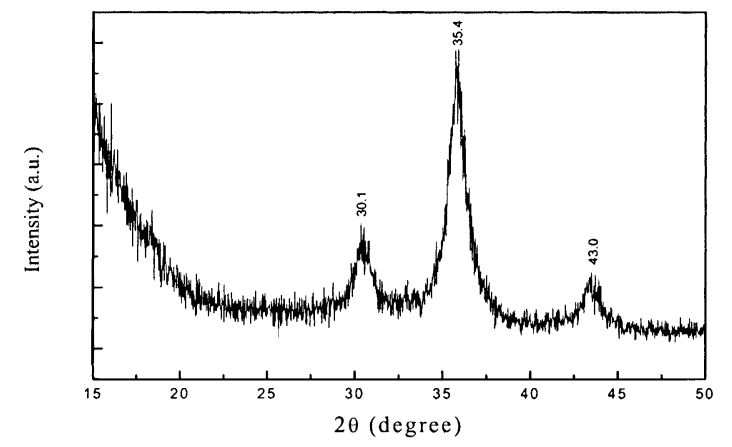

Fig. 1. The X-ray diffraction patterns of magnetite sample.

Fig. 2 illustrates the TEM micrograph of the PAA-coated magnetite. The magnetite displayed an uniform particle morphology and each particle was approximately $10 \mathrm{~nm}$ in diameter. The size characteristics of the PAA stabilized magnetite were also examined by dynamic light scattering (DLS). Fig. 3 reveals a fairly narrow size distribution and an average hydrodynamic diameter of approximately $93 \mathrm{~nm}$. Notably, the size of $\mathrm{Fe}_{3} \mathrm{O}_{4}$ depended somewhat on the method employed. The data from TEM was comparable to that of from XRD, but much smaller than the DLS results, possibly owing to that the water-soluble PAA chains were loosely bound on $\mathrm{Fe}_{3} \mathrm{O}_{4}$ and fully extended by dispersion medium and ultimately decreasing the diffusion coefficient of magnetite particle substantially.

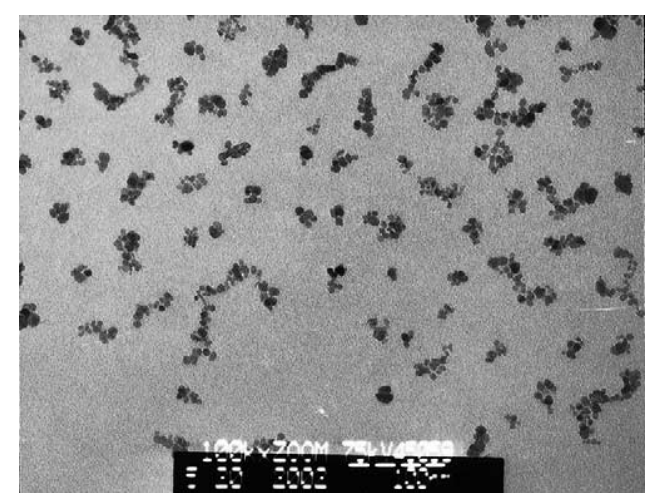

Fig. 2. TEM micrograph of magnetite nanoparticles

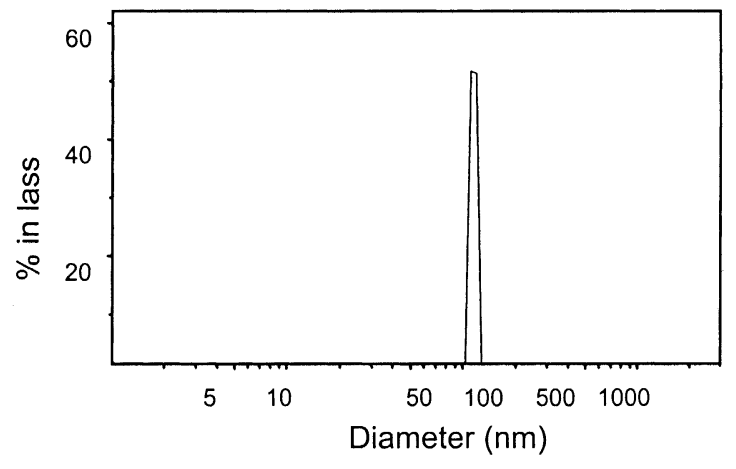

Fig. 3. Particle size distribution of PAA-coated $\mathrm{Fe}_{3} \mathrm{O}_{4}$ ferrofluid .

Fig. 4 presents the magnetization of bare and PAA-coated $\mathrm{Fe}_{3} \mathrm{O}_{4}$ nanoparticles as a function of applied field at $15 \mathrm{~K}$. When a magnetic field was applied, the dipolar particles aligned themselves with the field, resulting in a measurable magnetization. From the hysteristic loop, there seems existing a ferromagnetic phase, but without saturation up to 20,000 Oe. Based on our M-T curve (not shown here), it is majorly super-paramagnetic. Furthermore, the magnetization of PAA-coated $\mathrm{Fe}_{3} \mathrm{O}_{4}$ nanoparticles was only slightly lower than that of $\mathrm{Fe}_{3} \mathrm{O}_{4}$ nanoparticles.

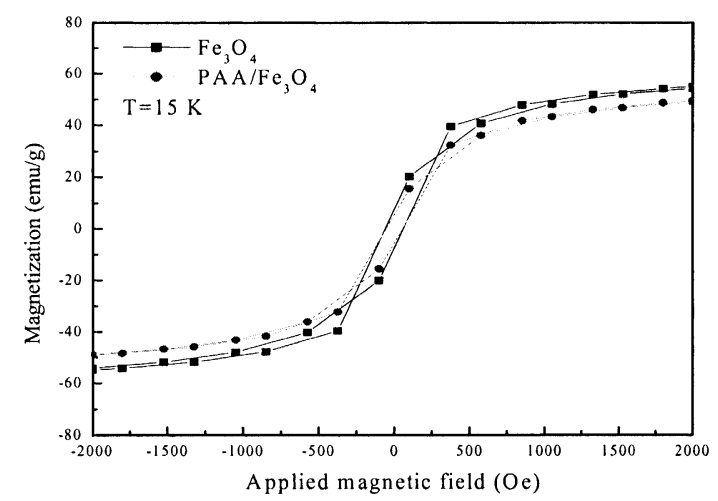

Fig. 4. Magnetization as a function of applied magnetic field at $\mathrm{T}=15 \mathrm{~K}$.

In conclusion, this work has described an efficient means of synthesizing a stable, water-based magnetic fluid consisting of PAA-coated $\mathrm{Fe}_{3} \mathrm{O}_{4}$ nanoparticles with a mixed phase of ferro- and superpara-magnetism.

\section{Acknowledgement}

The authors would also like to thank the National Science Council of the Republic of China for financially supporting this research under Contract No. NSC 89-2119-M-002-014.

\section{References}

[1] K. Ray and R.Casciari, J. Magn. Magn. Mater. 149 (1995) 174

[2] S. Risbud, Y. S. Kang, P. Stroeve, and J. F. Rabolt, Chem. Mater. 8 (1996) 2209.

[3] Sanaa E. Khalafalla, and George W. Reimers, IEEE Trans. Magn. MAG-16 (1980) 178.

[4] R. Massart, V. Cabuil, J. Phys. Chem. 84 (1984) 967. 